\title{
Quantum Hydrodynamic Model for the enhanced moments of Inertia of molecules in Helium Nanodroplets: Application to $\mathrm{SF}_{6}$
}

\author{
Kevin K. Lehmann 7 and Carlo Callegari \\ Department of Chemistry, Princeton University, Princeton NJ 08544 USA
}

(Dated: November 7, 2018)

\begin{abstract}
The increase in moment of inertia of $\mathrm{SF}_{6}$ in helium nanodroplets is calculated using the quantum hydrodynamic approach. This required an extension of the numerical solution to the hydrodynamic equation to three explicit dimensions. Based upon an expansion of the density in terms of the lowest four Octahedral spherical harmonics, the predicted increase in moment of inertia is $170 \mathrm{u} \AA^{2}$, compared to an experimentally determined value of $310(10) u \AA^{2}$, i.e., $55 \%$ of the observed value. The difference is likely in at least part due to lack of convergence with respect to the angular expansion, but at present we do not have access to the full densities from which a higher order expansion can be determined. The present results contradict those of Kwon et al., J. Chem. Phys. 113, 6469 (2000), who predicted that the hydrodynamic theory predicted less than $10 \%$ of the observed increase in moment of inertia.
\end{abstract}

*Electronic address: Lehmann@princeton.edu 
There is considerable current interest in the spectroscopy of atoms and molecules solvated in liquid Helium, particularly in ${ }^{4} \mathrm{He}$ nanodroplets [1]. These provide microscopic probes of the nature of this unique substance, the only physical system whose equilibrium state remains a liquid as $T \rightarrow 0 \mathrm{~K}$. Further, helium has properties that make it an almost ideal 'matrix' for the production and characterization of novel chemical species [2]. One such property is that even highly anisotropic solutes give rotationally resolved spectra, though with effective rotational moments of inertia several times that of the isolated molecule in the gas phase [3, 4]. Development of a quantitative predictive theory for the enhanced effective moments of inertia will be valuable for future use of the spectroscopically observed rotational constants to help determine the chemical carrier of an unassigned spectral feature, as they are often used in gas phase spectroscopy.

It has been widely recognized that enhanced moments of inertia arise from the kinetic energy of helium motion that is correlated with the rotation of the solute. In the case of $\mathrm{SF}_{6^{-}}$ $\mathrm{He}_{N}$ [5], a fixed frame, fixed node diffusion Monte Carlo calculations on small clusters with $N=8-20$ He atoms have recovered rotational excitation spectra in excellent agreement with that observed for $\mathrm{SF}_{6}$ in $\mathrm{He}$ nanodroplet with much larger number of helium atoms. Such calculations do not directly give any dynamical information, however, and thus leave open the question of how to physically characterize the helium motion. Two quantitatively predictive dynamical models have been put forth that invoke very distinct types of helium motion [6, 8]. In one [6, 7], a proposed 'nonsuperfluid fluid' density of helium was calculated using Path Integral Monte Carlo (PIMC) methods, and this density is assumed to rotate rigidly with the molecule provided that the molecule-He potential is sufficiently anisotropic relative to the induced rotational energy. To date, this two fluid model has been applied only to $\mathrm{SF}_{6}$ and OCS [6, 7]. For $\mathrm{SF}_{6}$, the 'two fluid model' gave a result in excellent agreement with experiment [3]. The predicted increase in the effective moment of inertia, $\Delta I$, was calculated as $327 \mathrm{u} \AA^{2}$ compared to a valued of $310(10) \mathrm{u} \AA^{2}$ calculated from the observed rotational constants. The level of agreement, fact, is far better than should be expected given the considerably uncertainty in the relevant $\mathrm{He}_{-} \mathrm{SF}_{6}$ interaction potential [9]. The two parameters that determine the anisotropy of the $\mathrm{He}_{-} \mathrm{SF}_{6}$ potential well have reported values of $-0.6 \pm 0.3$ and $0.14 \pm 0.14$ in Ref. [9].

The second approach, published by ourselves and coworkers [8], is based upon a hydrodynamic treatment for the helium flow, which is assumed to maintain a constant solvation 
density in the frame rotating with the molecule and be ideal (aviscous and irrotational). The hydrodynamic approach was applied to a number of linear molecules, and was found to be in good agreement for heavier molecules, including OCS. A key assumption of this work is that of adiabatic following by which we mean that the helium density in the molecular frame of a rotating molecule is the same as for a rigid molecule, fixed in the laboratory frame. It has recently been established experimentally [10] that for lighter molecules, in particular HCN and DCN, that adiabatic following breaks down and explains at least the sign of the error of the hydrodynamic theory in these cases.

In order to apply the hydrodynamic model, one needs the three dimensional helium density around the solute, which is known to be highly structured. The earlier hydrodynamics work used helium density functional theory (DF) [11] to estimate this quantity. For systems with cylindrical or higher symmetry, DF is many orders of magnitude computationally less expensive than Quantum Monte Carlo Methods but introduces additional uncertainties beyond the ever present uncertainty in the Helium-Solute interaction potential. In this work, the helium density around a static $\mathrm{SF}_{6}$ molecule, previously published by Barnett and Whaley [12], is used to calculate the hydrodynamic contribution to the moment of inertia of this molecule. Because of the different symmetry $\left(\mathrm{O}_{\mathrm{h}}\right.$ versus $\left.\mathrm{C}_{\infty \mathrm{v}}\right)$ of this solute compared to those treated previously, some changes had to be made in the computational procedure. Most significant, one degree of freedom could not be removed by separation of variables (as it could be for a cylindrically symmetric density) and, as a result, the hydrodynamic equation for the velocity potential had to be solved numerically in three degrees of freedom. The computational procedures used in the present case are described in the next section and the results presented in the last section.

\section{HYDRODYNAMIC CALCULATIONS}

The hydrodynamic calculations require an estimate of the three dimensional helium density around the solute molecule, $\mathrm{SF}_{6}$ in this case. This density will be totally symmetric in the point group of the molecule, $\mathrm{O}_{\mathrm{h}}$ in this case. This density, $\rho(r, \Omega)$, can be expanded in terms of the spherical tensor operators:

$$
\rho(r, \Omega)=\rho_{0}(r)+a_{4}(r) T_{4}(\Omega)+a_{6}(r) T_{6}(\Omega)+a_{8}(r) T_{8}(\Omega)+\ldots
$$


where $\Omega=(\theta, \varphi)$ and $T_{L}$ are linear combinations of the spherical harmonics, $Y_{L M}$ 's, that transform as $A_{1 \mathrm{~g}}$ in the $O_{\mathrm{h}}$ point group. In particular:

$$
\begin{aligned}
T_{4} & =\sqrt{\frac{7}{12}} Y_{40}+\sqrt{\frac{5}{24}}\left(Y_{40}+Y_{4-4}\right) \\
T_{6} & =\sqrt{\frac{1}{8}} Y_{60}-\frac{\sqrt{7}}{4}\left(Y_{60}+Y_{6-4}\right) \\
T_{8} & =\frac{\sqrt{33}}{8} Y_{80}+\frac{\sqrt{42}}{24}\left(Y_{84}+Y_{8-4}\right)+\frac{\sqrt{390}}{48}\left(Y_{88}+Y_{8-8}\right)
\end{aligned}
$$

Fox and Ozier [13] have presented a general procedure for to calculate these and higher harmonics.

Barnett and Whaley have calculated the helium density around $\mathrm{SF}_{6}$ using the diffusion Monte Carlo method. Their paper contains figures which give the radial isotropic density, $\rho_{0}(r)$, and radial 'cuts' of the density along the $\mathrm{C}_{2}, \mathrm{C}_{3}$, and $\mathrm{C}_{4}$ symmetry axes. If we assume that the density contains only the terms explicitly given in Eq. 1, then we can use the isotropic density and the density cuts to determine the radial coefficients $a_{4}(r), a_{6}(r)$, and $a_{8}(r)$ by using the equations:

$$
\begin{aligned}
\sqrt{\frac{21}{\pi}} a_{4}(r) & =\frac{378}{143} \rho_{0}-\frac{256}{143} \rho_{2}-\frac{378}{143} \rho_{3}+\frac{256}{143} \rho_{4} \\
\sqrt{\frac{26}{\pi}} a_{6}(r) & =-\frac{20}{55} \rho_{0}-\frac{128}{55} \rho_{2}+\frac{108}{55} \rho_{3}+\frac{40}{55} \rho_{4} \\
\sqrt{\frac{561}{\pi}} a_{8}(r) & =-\frac{1680}{65} \rho_{0}+\frac{768}{65} \rho_{2}+\frac{432}{65} \rho_{3}+\frac{480}{65} \rho_{4}
\end{aligned}
$$

In these equations, $\rho_{i}$ represents the radial density along the $\mathrm{C}_{i}$ symmetry axis. The radial density functions where determined by digitization of the images from the paper by Barnett and Whaley for clusters of 69 Helium atoms, the largest for which they reported the density cuts. These equations were derived by evaluation of the tensor operators $\left(T_{i}\right)$ along each of the symmetry axes and then inverting the linear system that relates the calculated density along each cut. Figure 1 shows the radial tensor densities calculated from these density cuts. It is apparent from this figure that the density in the first solvation shell (which is the most important for determination of the moment of inertia) is likely not fully converged with the present truncation of the density expansion. Unfortunately, we were not able to obtain the primary data from reference [12] which would have allowed the expansion to be carried to higher order. 
The assumption that the helium motion is irrotational $(\boldsymbol{\nabla} \times \mathbf{v}=0)$ implies that the velocity, $\mathbf{v}$, can be written as the gradient of a scalar function, $\mathbf{v}=-\boldsymbol{\nabla} \phi$, where $\phi$ is known as the velocity potential. In order to calculate the increased moment of inertia caused by motion of the helium, we will assume that the molecule is undergoing classical rotation with angular velocity $\omega$ around the $z$ axis. The equation of continuity gives the following equation:

$$
\boldsymbol{\nabla} \cdot(\rho \boldsymbol{\nabla} \phi)=\frac{\partial \rho}{\partial t}=-(\boldsymbol{\nabla} \rho) \cdot(\omega \hat{\boldsymbol{\varphi}})
$$

where the second equality comes from the assumption that the helium density remains constant in the frame rotating with angular velocity $\omega$ around the $z$ axis. The boundary conditions on $\phi$ are that the normal component of the fluid velocity match the normal component of velocity of any surface. The solution to Eq. 8 is linear in the angular velocity and thus is solved numerically for unit velocity. Equating the helium kinetic energy with the rotational kinetic energy, we can define $\Delta I_{\mathrm{h}}$ by:

$$
\begin{aligned}
\frac{1}{2} \Delta I_{\mathrm{h}} \omega^{2} & =\frac{1}{2} m_{\mathrm{He}} \int \rho|\nabla \phi|^{2} \mathrm{~d} V \\
& =\frac{1}{2} m_{\mathrm{He}}\left[-\int \phi\left(\frac{\partial \rho}{\partial t}\right) d V+\int \rho \phi(\boldsymbol{\nabla} \phi) \cdot d \mathbf{S}\right]
\end{aligned}
$$

The first equation holds for any velocity potential. The second has been derived from the first using vector identities and Eq. 8. As such, these two estimates need only be equal for $\phi$ that is a solution to Eq. 8. It can also be shown that the net orbital angular momentum produced by the helium flow is, for the solution of Eq. 8, $\mathbf{J}_{\mathrm{He}}=\Delta I_{\mathrm{h}} \boldsymbol{\omega}$.

A numerical solution for $\phi$ was found on a grid of points in a spherical coordinate system. The three cartesian axes of this coordinate system is aligned with three of the S-F bonds. The range of $r$ was selected between 3.5 and $10 \AA$ with between 51 and 201 radial points. The normal component of $\phi$ was selected to be zero on both the inner and outer constant $r$ surfaces. $\phi$ must be invariant to reflection in the $x, y$ plane, and thus we numerically restricted the solution to the $\theta=[0, \pi / 2]$ and restrict the solution to have zero normal derivative on the $x, y$ plane. Reflection in each of the four planes perpendicular to the $z$ axis is equivalent to change in the direction of rotation, and thus leads to a change in the sign of $\phi$. As a result, the numerical solution for $\phi$ could be restricted to the domain $\varphi=[0, \pi / 4]$ and $\phi$ selected to be zero on each of the planes $\varphi=0$ and $\pi / 4$. Between 41 and 161 equally spaced angular points were used in each of the angular coordinates coordinates. Since $\phi$ or 
its normal derivative is equal to zero on all the boundary surfaces, the surface integral in Eq. 10 is zero.

The inhomogeneous partial differential Eq. 8 was converted to a finite difference equation in the grid of points and solved by Gauss-Seidel iteration with successive overrelaxation [14. These equations (which involve $\ln \rho$ and its gradient) become singular when $\rho=0$. As a result, the density is bounded to remain above a threshold value, which was selected as $\rho_{\text {min }}=10^{-5} \AA^{-3}$ in this work. Solutions were iterated until the mean squared change in $\phi$ on the grid points was less than a fixed fraction $\left(10^{-10}\right.$ in this work) of the mean squared value of $\phi$ on the grid points. For the largest grid used, this required about 6000 iteration cycles with an overrelaxation parameter of 1.4 ( $\omega$ in the notation used in Numerical Recipes [14]). The two integral estimates for $\Delta I_{\mathrm{h}}$ are found to be 171 and $168 \mathrm{u} \AA^{2}$ respectively. This can be compared with a value of $\Delta I=310(10) \mathrm{u} \AA^{2}$ inferred from the observed effective rotational constant of $\mathrm{SF}_{6}$ in ${ }^{4} \mathrm{He}$ nanodroplets [3].

Kwon and Whaley [6] reported the 'superfluid' densities along the same symmetry axes, as calculated using PIMC and their proposed superfluid estimator. These have greatly decreased anisotropy compared to the total density. Repeating the hydrodynamic calculation using these densities gave $\Delta I_{\mathrm{h}}=12 \mathrm{u}^{2}$. This can be compared to their 'superfluid' contribution of the moment of inertia increase of $22 \mathrm{u} \AA^{2}[7]$. These authors also claim to have calculated the hydrodynamic contribution to the rotational constant used the total density around $\mathrm{SF}_{6}$ and report $\Delta I_{\mathrm{h}}=31 \mathrm{u} \AA^{2}$. This value is 5.5 times smaller than the value we have calculated. Based upon our extensive experience with similar hydrodynamic calculations, the modest $50 \%$ in hydrodynamic moment of inertia is inconsistent with the considerably greater angular anisotropy of the total density compared to their reported 'superfluid' density.

\section{DISCUSSION}

The hydrodynamic prediction for the enhanced moment of inertia of $\mathrm{SF}_{6}$ in helium is $\sim 55 \%$ of the experimentally observed value. This can be contrasted with the results reported in [8], where for heavier rotors, the theory appeared to systematically overestimate the size of the increased moment of inertia. Similarly, an overestimate of the hydrodynamic estimate for the increased moment of inertia was found for a model problem of a planer 
rotor interacting with a rigid ring of He atoms [15]. One explanation for the underestimate in the present case is that the density model used has underestimated the true anisotropy of the helium density, particularly in the first solvation shell where the hydrodynamic kinetic energy density is highest. This is supported by the results presented in fig 1, where it is demonstrated that the highest order anisotropy we have retained is still of considerable size in this first shell. Since the $\varphi$ derivative of the density is the source term in the hydrodynamic equation, addition of higher order anisotropies is expected lead to increased hydrodynamic motion and thus an increased estimate of $\Delta I_{\mathrm{h}}$. This explanation for the limited success of the hydrodynamic model in the present case can be tested by repeating the hydrodynamic calculation using the full anisotropic helium density calculated by Quantum Monte Carlo methods.

An alternative explanation, which cannot be ruled out at present, is that some fraction of the helium motion is not 'irrotational'. The Kelvin minimum energy principle [16] states that any 'rotational' solution to the equation of continuity will generate higher helium kinetic energy and thus He contribution to the moment of inertia. This would include the hydrodynamics assumed by the 'two fluid' model of Kwon and Whaley [6].

The fixed node, Diffusion Monte Carlo calculations of Lee et al. [5] were in excellent agreement with the experimental rotational excitation energies. This suggest that, barring cancelation of errors, that the nodal structure of the wavefunction assumed in that work (which had the nodal properties of the rigid rotor wavefunction for the $\mathrm{SF}_{6}$ ) should be a reasonable description of the true many body wavefunction for this system. It is useful, therefore, to examine how consistent the hydrodynamic model is with the functional form assumed in that work.

In a future publication, we will present a quantum derivation of the hydrodynamic approach. In that work, it will be shown that Eq. 8 arises from a variational optimization of a one particle phase function that multiples the ground state wavefunction that describes the helium in the frame that rotates with the molecule. The wavefunction for the orientation of this axis system in space will be the rigid rotor function for the molecule. Thus, the presence of the one particle phase functions will modify the nodal surfaces from that assumed in the Lee et al. calculation. The size of the one particle phase argument will be $m_{\mathrm{He}} \phi / \hbar$. The maximum value for $\phi$ for our solution is $\sim 5 \AA^{2} \omega$, and for the $J=1$ level we can approximate $\omega=4 \pi B_{\text {eff }}$, where $B_{\text {eff }}$ is the effective moment of inertia of $\mathrm{SF}_{6}$ in helium, 
$1.04 \mathrm{GHz}$. This gives a maximum hydrodynamic phase of $\approx 0.04$, and thus it appears that this will lead of small changes in the nodal properties. We would like to point out that for the model problem of the planer rotor coupled to a ring of Helium (which can of course be solved exactly), the errors in the equivalent fixed node approximation was of this same size and yet the DMC estimate of the rotational excitation energy had errors of at most a few percent.

\section{ACKNOWLEDGEMENT}

The authors wish to acknowledge Prof. Giacinto Scoles for many helpful discussions. This work was supported by the National Science Foundation and the Air Force Office of Scientific Research.

[1] J. P. Toennies and A. F. Vilesov, Annual Reviews of Physical Chemistry 49, 1 (1998).

[2] K. K. Lehmann and G. Scoles, Science 279, 2065 (1998).

[3] M. Hartmann, R. E. Miller, J. P. Toennies, and A. F. Vilesov, Physical Review Letters 95, $1566(1995)$.

[4] C. Callegari, A. Conjusteau, I. Reinhard, K. K. Lehmann, and G. Scoles, Journal of Chemical Physics 113, 10535 (2000).

[5] E. Lee, D. Farrelly, and K. B. Whaley, Physical Review Letters 83, 3812 (1999).

[6] Y. Kwon and K. B. Whaley, Physical Review Letters 83, 4108 (1999).

[7] Y. Kwon, P Huang, M. V. Patel, D. Blume, and K. B. Whaley, Journal of Chemical Physics 1136469 (2000).

[8] C. Callegari, A. Conjusteau, I. Reinhard, K. K. Lehmann, G. Scoles and F. Dalfovo, Physical Review Letters 83, 5058 (1999); 84, 1848 (2000).

[9] R. T. Pack, E. Piper, G. A. Pfeffer, and J. P. Toennies, Journal of Chemical Physics 80, 4940 (1983).

[10] A. Conjusteau, C. Callegari, I. Reinhard, K. K. Lehmann, and G. Scoles, Journal of Chemical Physics 113, 4840 (2000).

[11] M. Casas et al., Zeitschrift für Physik D 35, 67 (1995). 


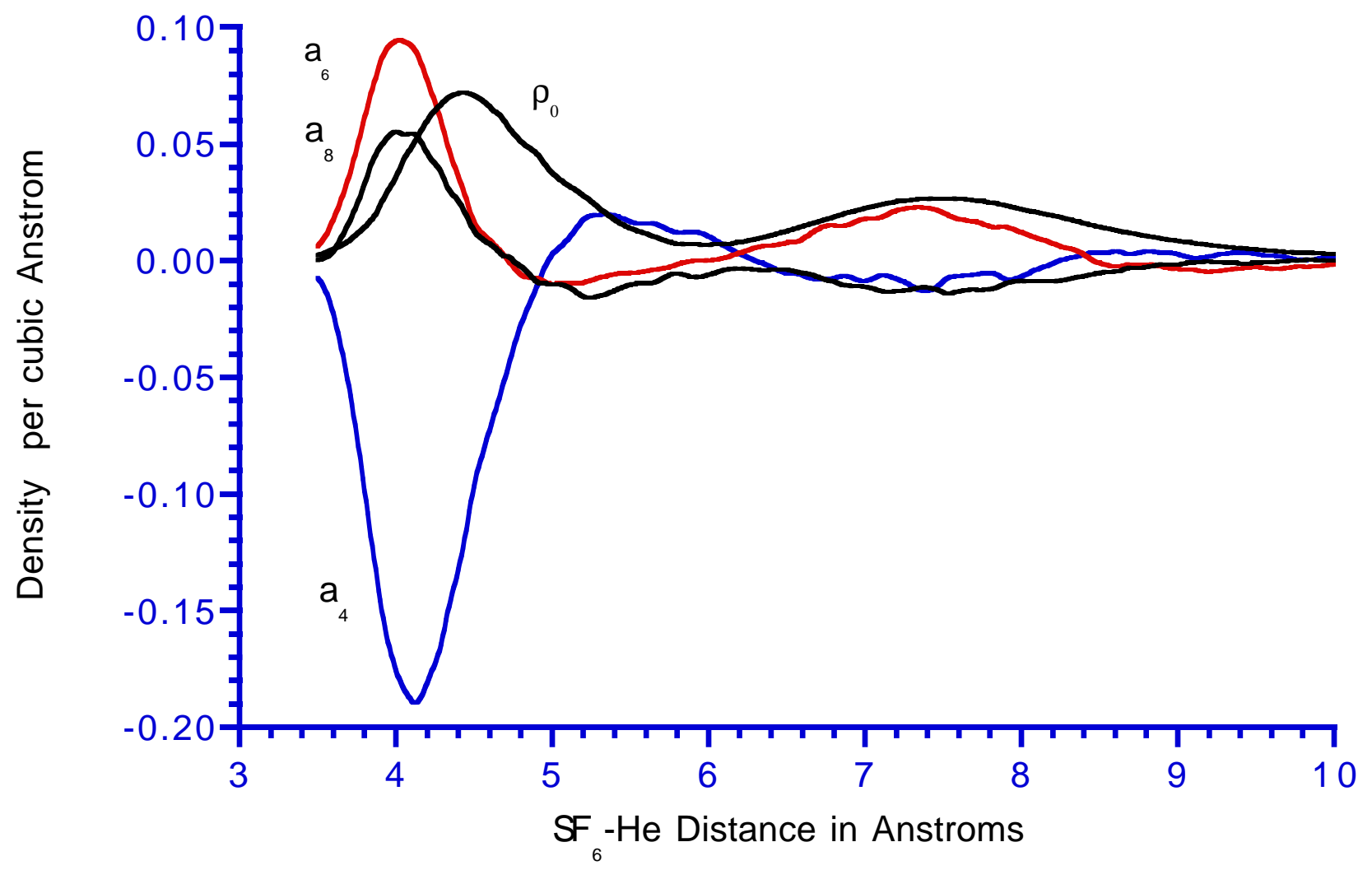

FIG. 1: The tensor densities, $\rho_{0}(r), a_{4}(r), a_{6}(r)$, and $a_{8}(r)$, in $\AA^{-3}$, as a function of the He-SF 6 radial distance in $\AA$

[12] R. N. Barnett and K. B. Whaley, Journal of Chemical Physics 99, 9730 (1993).

[13] K. Fox and I. Ozier, Journal of Chemical Physics 52, 5044 (1970).

[14] W. H. Press, B. P. Flannery, S. A. Teukolsky, and W. Vetterling, Numerical Recipes (Cambridge University Press, Cambridge, 1986).

[15] K. K. Lehmann, Journal of Chemical Physics 114, 4643 (2001).

[16] L. M. Milne-Thomson, Theoretical Hydrodynamics, fifth ed. (Dover, New York, 1996). 